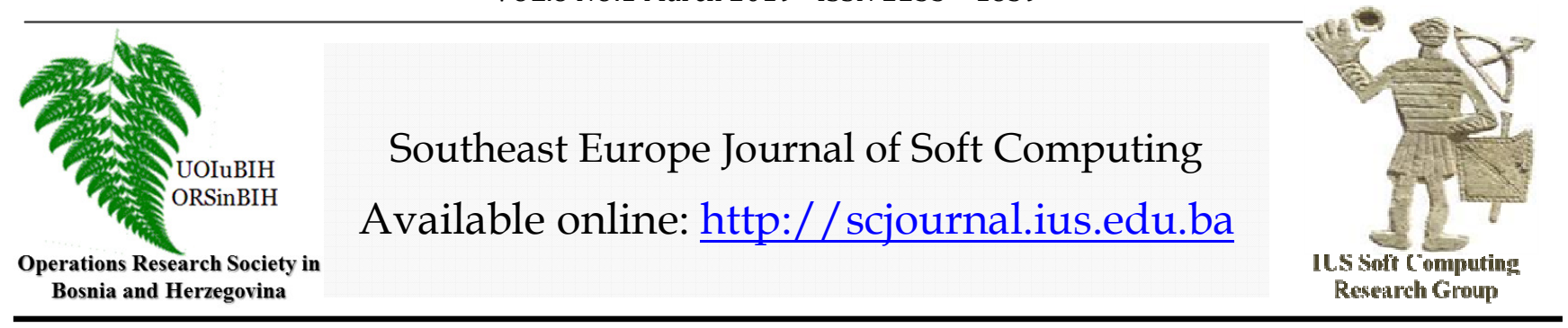

\title{
Zeka - Friendy Chatterbot
}

\author{
Rialda Spahić \\ Džana Bašić \\ Emine Yaman \\ Faculty of Engineering and Natural Sciences, \\ International University of Sarajevo International University of Sarajevo, \\ HrasnickaCesta 15, Ilidža 71210 Sarajevo, \\ Bosnia and Herzegovina \\ rialda.spahic@gmail.com \\ dzana_basic@hotmail.com \\ eyaman@ius.edu.ba
}

\section{Article Info}

Article history:

Article received on 10 January 2019

Received in revised form 1 February 2019

Keywords:

natural language processing, chatbots, artificial intelligence

\begin{abstract}
The idea of chatbots firstly appeared in the 1960s. But only after more than half a century passed the world became ready for their implementation into the real life, this being a result of the rapid progress in natural language processing, artificial intelligence, and the global presence of text messaging applications. Today, specialized chatbots exist in different domains, thus helping organizations handle large amount of inquiries. Idea of this project was to develop one friendly chatbot with whom you can talk about politics, movies, weather, sport, emotions and similar everyday things. Friendly chatbot named Zeka, is a web-based chatbot developed with the help of Chatterbot library. Chatbot relies on different natural processing and machine learning algorithms altered by its developers to increase its performance.
\end{abstract}

\section{INTRODUCTION}

Dialogue systems and conversational agents - including chatbots, personal assistants and voice control interfaces are becoming ubiquitous in modern society. Examples of these include personal assistants on mobile devices, technical support help over telephone lines, as well as online bots selling anything from fashion clothes and cosmetics to legal advice and self-help therapy. However, building 100\% intelligent conversational agents remains a major unsolved problem in artificial intelligence research [1]. A chatbot (also known as a talkbot, chatterbot, Bot, IM bot, interactive agent, or Artificial Conversational Entity) is defined to be a computer program which conducts a conversation in natural language via auditory or textual methods, understands the intent of the user, and sends a response based on business rules and data of the organization [2]. According to research done in 2016, $80 \%$ of businesses operating in United States plan to adopt at least one chatbot by the end of 2020 [2]. As this represents an increasing trend in today's world, many developers try to bring their own contribution to building a powerful AI tools and libraries that would enable a chatbot to easily conduct everyday conversations and thus pass the Turing test [3]. Idea of our project was to research the most recent AI libraries that are used for development of this kind of intelligent programs. Socialbot Zeka, developed in Python, is based on a large-scale ensemble system leveraging machine learning and natural language processing algorithms. Altering the existing set of algorithms and improving their performance, we were able to provide more sense to chatbot in terms of retrieving more common and reliable answers. These models are combined into an ensemble, which generates a candidate set of dialogue responses. Further, we applied intensive training to train the system to select an appropriate response from the models in its ensemble. Training is carried out on crowdsourced data and on interactions recorded between real-world users and a preliminary version of the system. Our bot 's interface is placed on web platform using Flask microframework for Python language. 
Chatbots are definitely a booming trend with more and more businesses and industries getting interested in it as they have eased the process of interaction. With the ever-increasing demand from consumers for personalization and human touch, the need for customer focused marketing is at its peak. The above-mentioned examples of chatbots are proving to be one ray of hope in making consumers happy and bridging the gap of human interaction. There is a lot that can be done to leverage more the potential of technologies like artificial intelligence, machine learning, and natural language processing.

\section{RELATED WORK}

In 1950, Alan Turing's famous article "Computing Machinery and Intelligence" was published, which proposed what is now called the Turing test as a criterion of intelligence [3]. This criterion depends on the ability of a computer program to impersonate a human in a real-time written conversation with a human judge, sufficiently well that the judge is unable to distinguish reliably on the basis of the conversational content alone between the program and a real human. The notoriety of Turing's proposed test stimulated great interest in Joseph Weizenbaum's program ELIZA [4], published in 1966, which seemed to be able to fool users into believing that they were conversing with a real human. ELIZA [4], was regarded as one of the first chatbots. ELIZA analysed input sentences and created its response based on reassembly rules associated with a decomposition of the input. This produced an impression of caring about its users, but it held no memory of the conversation and so could not enter into any form of targeted collaboration or negotiation. The syntactic language processing used by ELIZA has been developed significantly, leading to the development of a number of language processing chatbots.

A.L.I.C.E. [6] is a chatbot built using Artificial Intelligence Markup Language (AIML), developed over the course of 10 years. The chatbot is based on categories containing a stimulus, or pattern, and a template for the response. Category patterns are matched to find the most appropriate response to a user input. Further AIML tags provide for consideration of context, conditional branching and supervised learning to produce new responses. A.L.I.C.E. is a viable and experienced system but has not to our knowledge, as yet, been applied in a commercial environment. The Jabberwacky [7] chatbot had as its aim to "simulate natural human chat in an interesting, entertaining and humorous manner". Jabberwacky learns from all its previous conversations with humans. It functions by storing everything that is said to it, and uses contextual pattern matching techniques to select the most appropriate response. It has no hard-coded rules, instead relying entirely on previous conversations. It is explicitly not intended to do anything 'useful', instead being simply to chat [7].
Chatbots that appeared later, like Lingubot ${ }^{\mathrm{TM}}$ [11], offer sophisticated development environments allowing the building of intelligent conversational agents with complex, goal driven behaviour. In 'Lingubots' both the words and the grammatical structure of the user's input are analysed using customised templates. This facilitates the development of a user model, which is used in conjunction with the conversational context and specific words in the dialogue to determine the chatbot's response. Responses might include further conversation with the user, reading or writing to external systems (for instance to open a web page or update a database), or a combination of these. This rich range of responses allows for intelligent conversation with the user, and provides the ability to steer the user back to the task in hand if they stray from the designated discussion content for too long. Melody is medical chatbot launched in China [9]. It is designed to help doctors by facilitating faster flow of information between doctor and patient. Melody seeks to help patients by chatting with them and helping them to diagnose about their symptoms and gives a feedback to the real-life doctors. Many researchers asserted that it is the beginning of the AI-driven healthcare.

\section{CHATBOTS}

A chatbot (also known as a talkbot, chatterbot, Bot, IM bot, interactive agent, or Artificial Conversational Entity) is a computer program or an artificial intelligence which conducts a conversation via auditory or textual methods [1]. It is designed to perform a series of tasks on its own and without the help of a human being. Such programs are often designed to convincingly simulate how a human would behave as a conversational partner, thereby passing the Turing test [2]. Chatbots are typically used in dialog systems for various practical purposes including customer service or information acquisition. Tasks a bot can do can vary from things such as making a reservation at a restaurant, marking a date on the calendar or collecting and displaying information to its users, and informing the user about the weather, etc.

Some chatterbots use sophisticated natural language processing systems, but many simpler systems scan for keywords within the input, then pull a reply with the most matching keywords, or the most similar wording pattern, from a database. The term "ChatterBot" was originally coined by Michael Mauldin (creator of the first Verbot, Julia) in 1994 to describe these conversational programs. Today, most chatbots are either accessed via virtual assistants such as Google Assistant and Amazon Alexa, via messaging apps such as Facebook Messenger or WeChat, or via individual organizations' apps and websites.

There are two types of chatbots Rule based bots and AI bots.

\section{Rule-based Approach}

In a rule-based approach, a bot answers questions based on some rules on which it is trained on. The rules defined can be very simple to very complex. The creation of these bots is 
relatively straightforward using some rule-based approach, but the bot is not efficient in answering questions whose pattern does not match with the rules on which the bot is trained.

\section{AI-based Approach}

$\mathrm{AI}$ is the technology that allows the bot to learn from the interactions it has with the end users. Behind these learnings there are analytics platforms, and integrations with APIs, among other things, that feed the AI and provide resources so that that the chatbot is able to provide the user with correct answers.

Developed chatbot Zeka leans toward AI based chatbots, whose main goal is to engage into human-like conversation, and to provide user with the answers he seeks for.

\section{TECHNOLOGY}

As for the programming language of our chatbot we decided for Python, as one of the most popular languages [12], logical decision for the environment was PyCharm [13]. PyCharm is an environment that comes with a complete set of tools for Python development and provides capabilities for professional web development.

Developing chatbots using Python language is greatly made easier using ChatterBot [14], a Python library used for retrieving automated responses to a user's input. ChatterBot indeed uses a selection of machine learning algorithms to produce different types of responses. It includes tools that help simplify the process of training a chat bot instance. ChatterBot's training process involves loading example dialog into the chat bot's database. This either creates or builds upon the graph data structure that represents the sets of known statements and responses. When a chat bot trainer is provided with a data set, it creates the necessary entries in the chat bot's knowledge graph so that the statement inputs and responses are correctly represented.

Several training classes come built-in with ChatterBot. These utilities range from allowing you to update the chat bot's database knowledge graph based on a list of statements representing a conversation, to tools that allow you to train your bot based on a corpus of pre-loaded training data. Logic adapters determine the logic for how ChatterBot selects a response to a given input statement (Figure 1).

In order for our chatbot to operate efficiently it needs to rely on natural language processing and machine learning algorithms. Natural language processing (NLP) and Machine Learning are both fields in computer science related to AI. Machine learning can be applied in many different fields, and NLP takes care of "understanding" the natural language of the human that the program (e.g. chatbot) is trying to communicate with.

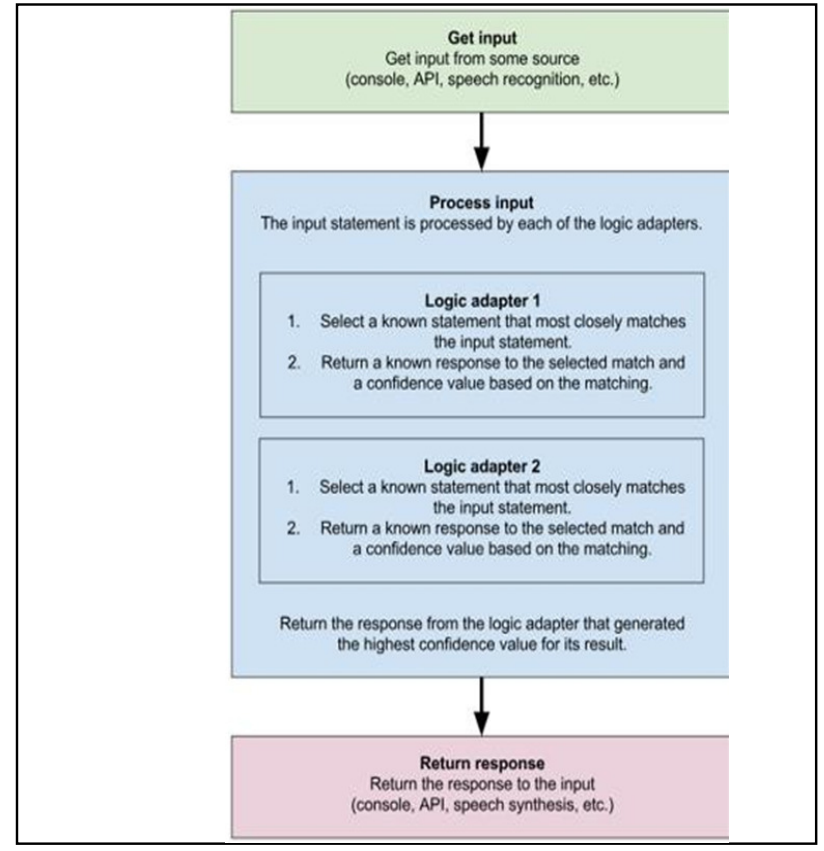

Figure 1 Process flow diagram of a Chatterbot

This understanding enables the program (e.g. chatbot) to both interpret input and produce output in the form of human language. It is easy to program a computer with the rules, but not easy to instruct it to notice new knowledge. Breaking down the language has become a science on its own. To properly understand each sentence means to understand it's context. This is done by extracting emotions, keywords and relationships from each sentence.

Another important feature is to present how the sentiments are analyzed in the input. It is important to take sentiments in observation because they are crucial to understanding the meanings of statements through context.

However, it is not enough to analyze single words, one by one, we need to analyze the full sentence.

If we read "I did not have a great day", all of the keywords are positive except the negation 'not', but the overall outcome is not positive (Figure 2). We need to look at the overall picture, beyond single word analysis, and this process is built-in inside the Chatterbot library. 


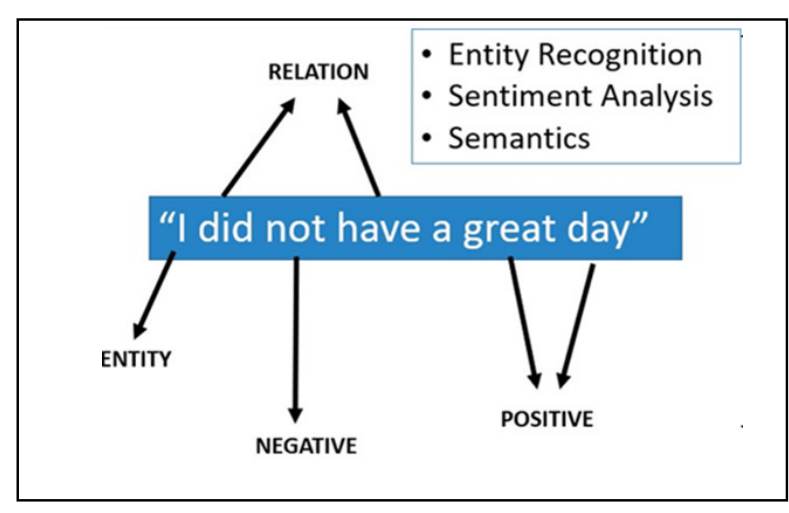

Figure 2 NLP Overview

\section{METHODOLOGY}

An example of typical input to the chatbot is depicted on Figure 3.

user: Good morning! How are you doing?

bot: I am doing very well, thank you for asking. user: You're welcome.

bot: Do you like hats?

Figure 3 Chatbot I/O Example

The way the chatbot retrieves responses is based on logical adapters selection on which Chatterbot is based on. The logic adapter that our bot uses are specified by setting the logic adapters parameter to the import path of the logic adapter. We decided for Best Match adapter, as it selects response based on the best-known match to a given statement.

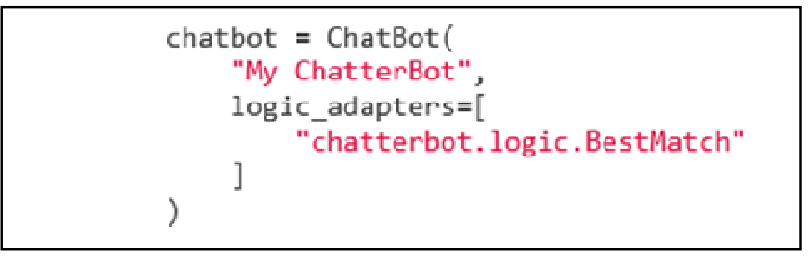

Figure 4 Logic adapters

After introducing best match operator to our bot, we also altered the database source that our bot is supposed to use. We created our own local database, where we firstly stored all the conversations the bot conducts, and later on that conversations are sent to centralized SQL storage.

Whenever our bot receives an input, in order to generate the output, best match adapter firstly looks into local storage, then visits centralized storage. The answer with highest confidence value is then returned as the output.

Loading the data from local storage first, enables the chatbot to give more accurate outputs as local conversations are more authentic to the particular user.

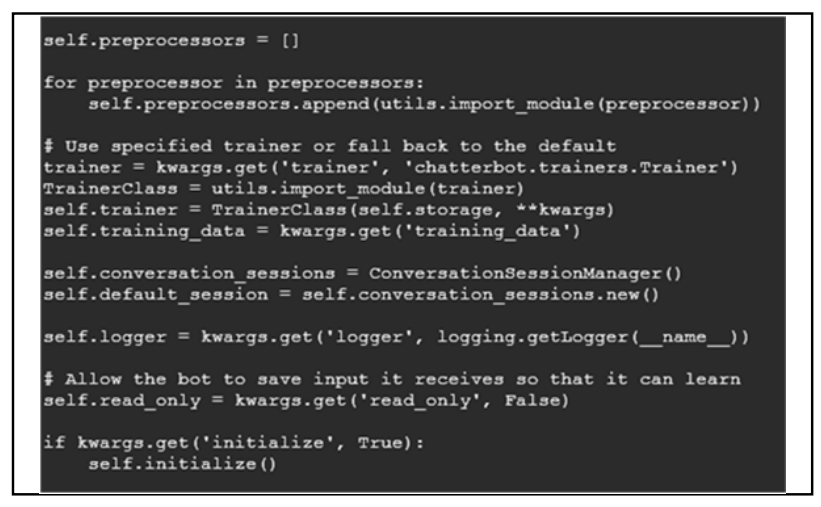

Figure 5 Trainer Modules

\section{RESULTS AND DISCUSSIONS}

We were able to integrate different conversational libraries such as: emotions, music, movies, weather, media, politics and everyday chat. Alongside, we have built a library on our own that would be 'fed' into Chatbot corpus for more convenient and realistic answers to input questions. It is not an easy task to perform human-like conversation with computing methods, therefore our program is prone to errors and obvious mistakes (Figure 6). Integrating chatbot into web-based application adds a value to the natural feel of conversation, such as those on common social media.

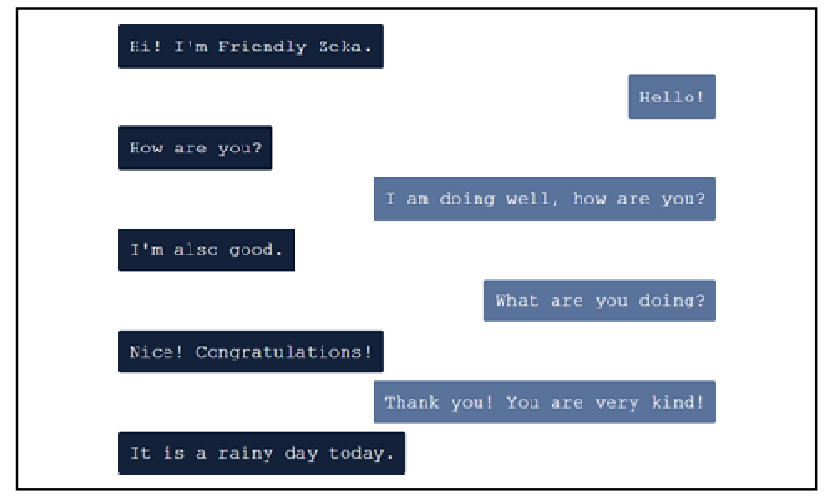

Figure 6 Friendly Zeka

However, meaningful conversations are obtained with training and continuous inputs of answers to the chatbot storage (Figure 7). 


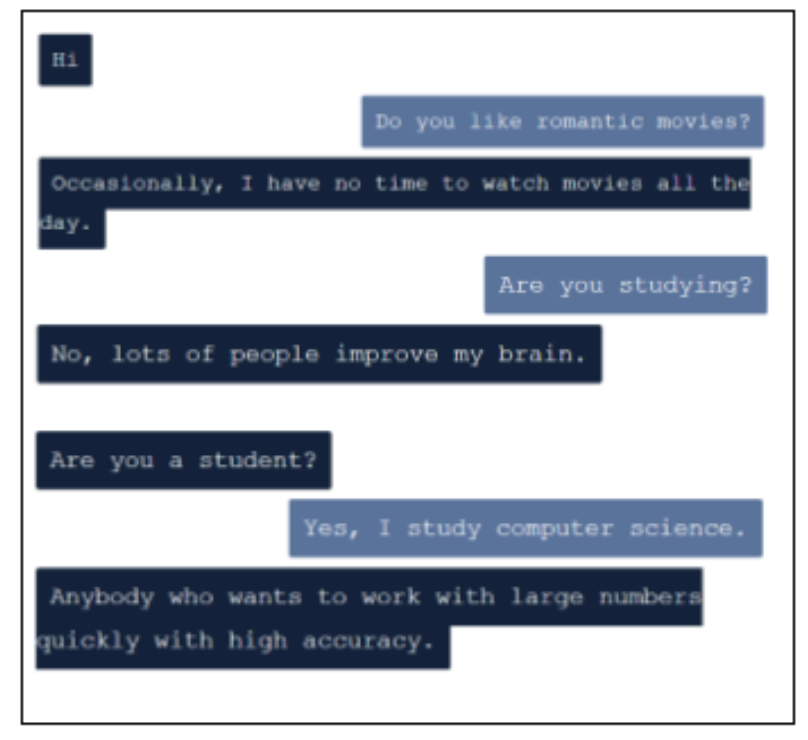

Figure 7 Conversation Flow

A received input message may be an explicit statement or unstructured content or activity in a textual form. The training material is a set of questions and responses ranked and grouped according to importance; variations of a set of questions and answers, information that are specific to an interest topic, such as with or without pre-trained responses; a text file of combinations of inputs and outputs and a set of questions from a centralized dictionary of questions. The chatbot answers questions from a variety of questions from different topics. It has a similarity with a number of social media chatters, which was the intent of the project. The majority of questions are directional or factual questions that Zeka can handle. The database proved to be practical to use and serve as a starting point to rebuild a similar corpus locally.

\section{CONCLUSION AND FUTURE WORK}

Artificial Intelligence is perhaps the most interesting as well as challenging field of research today. The aim of chatbot designers should be to build tools that help people, facilitate their work, and their interaction with computers using natural language but not to replace the human role totally, or imitate human conversation perfectly.

"We need not take human-human conversation as the gold standard for conversational exchanges. If one had a perfect simulation a human conversant, then it would be human human conversation and not human-computer conversation with its sometimes odd but pertinent properties." (Colby, 1999).

Our future work consists of extracting different news and social media APIs in order to extract meaningful and more natural conversations and store them into Chatbot corpus of knowledge. Another additional feature to be added is Speech-to-Text and Text-to-Speech functionality. This way, we would be a step closer to a more realistic and less errorprone conversation of human-computer.

\section{ACKNOWLEDGMENT}

We would like to express sincere gratitude to our professor Emine Yaman for guiding us through the field of Artificial Intelligence and presenting us the state-of-art of this field.

\section{REFERENCES}

[1] Chatbots, BRAIN (brn.ai) blockchain-based. "Chatbot Report 2018: Global Trends and Analysis - Chatbots Magazine." Chatbots Magazine, Chatbots Magazine, acceed: 17 Mar. 2018,

[2] Hald, Grasia. "Chatbot 101: Everything You Ever Wanted to Know about Chatbots." Medium, Augmenting Humanity, 15 Nov. 2017, medium.com/botsupply/chatbot-101everything-you-ever- wanted-to-know-about-chatbots$478 \mathrm{c} 0 \mathrm{~b} 825 \mathrm{dd} 0$.

[3] Turing, Alan M. "Computing machinery and intelligence." Parsing the Turing Test. Springer, Dordrecht, 2009. 23-65.

[4] Weizenbaum, Joseph. "ELIZA - a computer program for the study of natural language communication between man and machine." Communications of the ACM 9.1 (1966): 36-45.

[5] Artificial intelligence markup language (aiml). Resources avalaible at:

[6] http://alice.sunlitsurf.com/alice/aiml.html

[7] Wallace, Richard S. "The anatomy of ALICE." Parsing the Turing Test. Springer, Dordrecht, 2009. 181-210.

[8] Carpenter, R. 1997-2006. Jabberwacky.com. www.jabberwacky.com. Accessed 16/05/18

[9] "Nadia the Chatbot: The Rise of Emotionally Intelligent, Lifelike Customer Service Avatars." International Society for Presence Research, 27 Mar. 2017, ispr.info/2017/03/27/nadiathe-chatbot-the- rise-of-emotionally-intelligent-lifelikecustomer-service-avatars/.

[10] "Baidu's Melody." www.topbots.com/project/chinese- baidu-bot-ai-doctor/.

[11] Mailonline, Phoebe Weston For. "Facebook's Chatbot 'Dating Coach' Lara Sets up Singletons." Daily Mail Online, Associated Newspapers, 19 Apr. 2017, www.dailymail.co.uk/sciencetech/article- 4425658/Facebooks-chatbot-dating-coach-Lara-sets-singletons.html.

[12] Bramer, Max A., et al. Research and Development in Intelligent Systems XXVII Incorporating Applications and Innovations in Intelligent Systems XVIII Proceedings of AI2010, the Thirtieth SGAI International Conference on Innovative Techniques and Applications of Artificial Intelligence. Springer, 2011.

[13] Lobo, Joe, and Jordi Fontseca. "Choosing the Best Language to Build Your AI Chatbot." TechCrunch, TechCrunch, 20 Dec. 2017, techcrunch.com/2017/12/20/choosing-the-bestlanguage-to-build- your-ai-chatbot/.

[14] "PyCharm: Python IDE for Professional Developers by JetBrains." JetBrains, www.jetbrains.com/pycharm/.

[15] "About ChatterBot." About ChatterBot - ChatterBot 0.8.7 Documentation, chatterbot.readthedocs.io/en/stable/. 